\title{
FIXED-POINT-FREE ACTIONS ON A CLASS OF ABELIAN GROUPS
}

\author{
P. M. CURRAN
}

\begin{abstract}
It is proved that all cohomology groups of a group $G$ acting on an abelian group of a certain type vanish if the action of some subnormal abelian subgroup of $G$ is fixed-point-free. This result is then applied to obtain results about group extensions and about the complete reducibility of linear groups.
\end{abstract}

Introduction. The purpose of this paper is to prove the Proposition below and its corollary, Theorem 1, and to derive from the latter some consequences about group extensions and about the complete reducibility of linear groups. These results are contained in Corollaries 2-4. Statement (a) of $\$ 1$ and part (b) of the Lemma, \$2, are related to the surrounding material, but are not used in the subsequent proofs.

I am indebted to several colleagues for useful suggestions in connection with this paper. I am also grateful to the referee for his valuable comments, especially for pointing out that the original version of Theorem 1 could be strengthened to its present form.

In what follows, a $V^{1}$-module for a group $G$ will mean a finite direct sum $\sum V_{i}$, where $V_{i}$ is a finite-dimensional vector space over a field $k_{i}$, and $V_{i}$ is a $k_{i} G$-module. For $j \geqslant 2$, a $V^{j}$-module is a $G$-module $A$ for which there is a short exact sequence $0 \rightarrow A^{\prime} \rightarrow A \rightarrow A^{\prime \prime} \rightarrow 0$, where $A^{\prime}$ is a $V^{1}$-module and $A^{\prime \prime}$ is a $V^{j-1}$-module. $A$ is a $W$-module if it is a $V^{j}$-module for some $j$.

It can be shown that every $W$-module is a direct sum of a torsion group of finite exponent and a torsion free divisible group (i.e., a vector space over $\mathbf{Q}$ ). Conversely, one can show that every such abelian group is a $W$-module (for trivial group action). Moreover, if such a group $A=T \oplus F$ is given as a $G$ module, then $A$ is automatically a $W$-module for $G$ if the torsion part $T$ is finite and $F$ is finite dimensional over $\mathbf{Q}$. This follows by induction on $|T|$, the order of $T$, from the exact sequence

$$
0 \rightarrow S_{p} \rightarrow A \rightarrow A / S_{p}=T / S_{p} \oplus F \rightarrow 0,
$$

where $p$ is any prime dividing $|T|$ and $S_{p}=\{x \in A: p x=0\}$. (If $T=0$, the statement is clear.)

PROPOSITION. Let $G$ be a group, $N$ a subnormal abelian subgroup of $G, k a$

Received by the editors July 19, 1974 and, in revised form, February 25, 1975.

AMS (MOS) subject classifications (1970). Primary 18H10; Secondary 20F25, 20H20, $20 \mathrm{~J} 05$.

Key words and phrases. Group cohomology, group extensions, linear groups, complete reducibility, fixed-point-free actions.

๑ American Mathematical Society 1976 
field, and $V$ a $k G$-module, finite dimensional over $k$. If

$$
H^{0}(N, V)\left(=V^{N}=\{v \in V: h v=v \text { for all } h \in N\}\right)=0,
$$

then $H^{r}(G, V)=0$ for $r \geqslant 0$.

THEOREM 1. Let $A$ be a $W$-module for $G$. If $N$ is a subnormal abelian subgroup of $G$ such that $A^{N}=0$, then $H^{r}(G, A)=0$ for $r \geqslant 0$.

1. Preliminaries. Let $G$ be a group with center $C, R$ a commutative ring with unity $1 \neq 0$, and let $A$ be an $R G$-module. If $\alpha \in R C$, then the map $a \mapsto \alpha a$ is an $R G$-endomorphism of $A$. If $\varepsilon \alpha=0$, ( $\varepsilon$ is the unit augmentation), then the induced endomorphism $\alpha^{*}$ of each cohomology group $H^{r}(G, A)$ is the zero map. For if $X$ is an $R G$-projective resolution of the trivial $G$-module $R$, then the map $X \rightarrow X$, given by $x \mapsto \alpha x$, is a map over the zero map on $R$ (in the sense of $[1$, p. 76]), hence by [1, Chapter V, Proposition 1.1] is homotopic to the zero map $X \rightarrow X$. This homotopy carries over to the complex $\operatorname{Hom}(X, A)$, so the induced maps $\alpha^{*}$ and $0^{*}$ on the homology of this complex are the same. This remark is a special case of Exercise 6, Chapter X of [1], which can be proved in the same way.

Some consequences:

(a) If there exists $\alpha \in R C$ with $\varepsilon \alpha=0$ such that $\alpha$ is injective on $A$ (i.e., $a \mapsto \alpha a$ is an injective endomorphism of $A$ ) and there exists $\beta \in R G$ with $\varepsilon \beta=0$ such that $\beta$ is bijective on $A$, then $H^{1}(G, A)=0$.

(b) If there exists $\alpha \in R C$ with $\varepsilon \alpha=0$ such that $\alpha$ is bijective on $A$, then $H^{r}(G, A)=0$ for $r \geqslant 0$.

Proof. (a) The hypothesis implies that $H^{0}(G, A)=0$. Hence the long exact cohomology sequence associated with the exact sequence

$$
0 \rightarrow A \stackrel{\alpha}{\rightarrow} A \rightarrow A / A^{\prime} \rightarrow 0,
$$

where $A^{\prime}=\alpha A$, together with the fact that $\alpha^{*}=0$ by the italicized remark above, yields $H^{1}(G, A) \approx H^{0}\left(G, A / A^{\prime}\right)$. But if $a+A^{\prime} \in H^{0}\left(G, A / A^{\prime}\right)$, then $\beta\left(a+A^{\prime}\right)=0$, so $a+A^{\prime}=0$ since $\beta^{-1}$ commutes with $\alpha$.

(b) This is clear since $\alpha^{*}$ is an automorphism of $H^{r}(G, A)$ which is at the same time the zero map.

2. The main theorem. The proof of the Proposition will be based on part (a) of the following lemma. As the reader may easily see, part (b) could be used to give a more direct proof under the additional assumption that $\operatorname{dim} V<|k|$.

LEMMA. Let $B$ be a commutative subset of End $V, V$ a vector space of dimension $n<\infty$ over a field $k$ of order $q \leqslant \infty$, and suppose $V^{B}=\{v \in V: b v$ $=v$ for all $b \in B\}=0$.

(a) If $V$ is indecomposable as a B-module, then there exists $b \in B$ such that $b-1$ is invertible.

(b) If $n<q$, there exist $b_{i} \in B$ and $c_{i} \in k$ such that $\sum c_{i}\left(b_{i}-1\right)$ is invertible.

Proof. (a) For each $b \in B$, the minimum polynomial $\mu_{b}(X)$ is a power of an irreducible polynomial [3, Chapter IV, §9]. If $b \in B$ is such that $b-1$ is 
not invertible, it has 1 as an eigenvalue, so $\mu_{b}(X)=(X-1)^{r}$ for some $r$. Let $V^{\prime}$ be a $B$-irreducible subspace of $V$. Then the minimum polynomial of $b \mid V^{\prime}$ is irreducible [ibid.], hence is $X-1$. Thus, if no $b-1$ is invertible, then $V^{\prime} \subset V^{B}$, a contradiction.

(b) We write $V=V_{1} \oplus \cdots \oplus V_{r}$, with each $V_{i}$ indecomposable. By part (a), there exist $b_{j} \in B, j=1, \ldots, r$, such that $\left(b_{j}-1\right) \mid V_{j}$ is invertible. Relative to a suitable basis, the matrix $\beta\left(b_{j}\right)$ of $b_{j}$ is the direct sum of the matrices $\beta_{i}\left(b_{j}\right)$ of $b_{j} \mid V_{i}, i=1, \ldots, r$. Consider the matrix $\sum_{j=1}^{r} X_{j}\left(\beta\left(b_{j}\right)-1\right)$ in the ring of $n \times n$ matrices over the polynomial ring $k\left[X_{1}, \ldots, X_{r}\right]$, and let $f\left(X_{1}, \ldots, X_{r}\right)$ be its determinant. Then $f=\prod_{i=1}^{r} f_{i}$, where

$$
f_{i}\left(X_{1}, \ldots, X_{r}\right)=\operatorname{det} \sum_{j=1}^{r} X_{j}\left(\beta_{i}\left(b_{j}\right)-1\right) .
$$

Now, $f_{i} \neq 0$ since

$$
\begin{aligned}
f_{i}\left(0, \ldots, X_{i}, \ldots, 0\right) & =\operatorname{det} X_{i}\left(\beta_{i}\left(b_{i}\right)-1\right) \\
& =X_{i}^{\nu_{i}} \operatorname{det}\left(\beta_{i}\left(b_{i}\right)-1\right)
\end{aligned}
$$

$\left(\nu_{i}=\operatorname{dim} V_{i}\right)$, and this is nonzero by choice of $b_{i}$. Since $f \neq 0$, and the degree of $f$ in each $X_{i}$ is $\leqslant n<q$, there exist $c_{1}, \ldots, c_{r} \in k$ such that $f\left(c_{1}, \ldots, c_{r}\right)$ $\neq 0\left[4\right.$, Chapter $\mathrm{V}, \S 4$, Theorem 5], i.e., such that $\sum c_{j}\left(b_{j}-1\right)$ is invertible.

Proof of Proposition. We will show first that $H^{r}(N, V)=0$ for $r \geqslant 0$. We may assume that $V$ is $N$-indecomposable since the functor $H^{r}(N$, preserves direct sums.

First, apply part (a) of the Lemma with $B=\rho(N)$, where $\rho$ is the representation of $G$ in Aut $V$ corresponding to the given action of $G$ on $V$, to obtain $b \in B$ such that $b-1$ is invertible. Choose $h \in N$ so that $\rho(h)=b$ and apply statement (b) of $\S 1$ with $G=C=N, A=V$ and $\alpha=h-1$ to conclude that

$$
H^{r}(N, V)=0, \quad r \geqslant 0 .
$$

Since $N$ is subnormal, there is a series

$$
N=G_{0} \triangleleft G_{1} \triangleleft \cdots \triangleleft G_{s}=G .
$$

By (*) we have $H^{r}\left(G_{i}, V\right)=0$ for $i=0$. Now use the inflation-restriction sequence [6, Chapter VII, §6, Proposition 5]

$$
0 \rightarrow H^{r}\left(G_{i} / G_{i-1}, V^{G_{i-1}}\right) \rightarrow H^{r}\left(G_{i}, V\right) \rightarrow H^{r}\left(G_{i-1}, V\right)
$$

and the fact that $V^{G_{i-1}} \subset V^{N}=0$, to conclude inductively that $H^{r}\left(G_{i}, V\right)$ $=0, i=0, \ldots, s$.

Proof of Theorem 1. For $V^{1}$-modules, the statement is an immediate consequence of the Proposition. Now let $A$ be a $V^{j}$-module with $j \geqslant 2$ : $0 \rightarrow A^{\prime} \rightarrow A \rightarrow A^{\prime \prime} \rightarrow 0$. We have $0=A^{N} \rightarrow A^{\prime \prime N} \rightarrow H^{1}\left(N, A^{\prime}\right)=0$, so inductively $H^{r}\left(G, A^{\prime \prime}\right)=0$ for $r \geqslant 0$. Now the long exact sequence yields $H^{r}(G, A)=0$ for $r \geqslant 0$. 
$A^{N}=0$ ). If $A^{N}$ is a direct summand of $A$ (as a $W$-module for $G$ ), then

$$
H^{r}(G, A) \approx H^{r}\left(G, A^{N}\right) \text { for } r \geqslant 0 .
$$

Proof. If $A=A^{N} \oplus A_{1}$, then $A_{1}^{N}=0$, so $H^{r}\left(G, A_{1}\right)=0$.

RemarK. The hypothesis of Corollary 1 is automatically satisfied if $A$ is a finite-dimensional vector space, $N$ is normal in $G$ and $\rho(G)$ is completely reducible; e.g., if $A$ has a positive definite scalar product and $\rho(G)$ consists of orthogonal transformations.

3. Group extensions. We recall that any extension of a group $K$ by a group $G$ induces a coupling of $G$ to $K$, i.e., a homomorphism $G \rightarrow$ Out $K$ $=$ Aut $K / \operatorname{In} K$, where In $K$ is the group of inner automorphisms of $K$ [2, §5.1], and this induces an action of $G$ on the center of $K$. However, not every coupling gives rise to an extension.

We shall call an abelian group a $V$-group if it is the direct sum of a finite group and a finite-dimensional vector space over $\mathbf{Q}$.

Corollary 2. Let $K$ be a group whose center $A$ is a $V$-group and let $\chi: G \rightarrow$ Out $K$ be a coupling such that the action on $A$ of some subnormal abelian subgroup $N$ of $G$ is fixed-point-free. (As noted in the Introduction, $A$ is automatically a $W$-module for $G$.) Then:

(a) There exists a unique (up to equivalence) extension $E$ of $K$ by $G$ which induces $x$.

(b) If $K=A$ is abelian, then $E$ splits over $A$, and the only automorphisms of $E$ which leave $A$ and $E / A$ elementwise fixed are the inner automorphisms induced by elements of $A$.

Proof. By Theorem $1, H^{r}(G, A)=0$ for $r \geqslant 0$. The vanishing of $H^{3}$ implies the existence of an extension giving rise to $\chi$ [2, Chapter 5, Proposition 2] and the vanishing of $H^{2}$ implies the uniqueness of this extension [2, Chapter 5, Theorem 2].

The first part of conclusion (b) is clear and the second part follows from the vanishing of $H^{1}$ [2, Chapter 3, Proposition 5].

COROllary 3. Let $E$ be an extension of $a V$-group $A$ by an abelian group. If the center of $E$ is disjoint from $A$, then $E$ splits over $A$.

Proof. Use Corollary 2(b) with $G=N=E / A$.

The following counterexample shows that the hypothesis of divisibility of $A / T(T=$ torsion part $)$ may not be omitted in Corollary 3. Let $E=\langle a\rangle$ * $\langle b\rangle$, where $a$ and $b$ have order 2, and let $A=[E, E]$. Then $A=\left\langle(a b)^{2}\right\rangle$, so all hypotheses are satisfied except divisibility of $A / T$, but $E$ contains no subgroup isomorphic to $E / A=\mathbf{Z}_{2} \oplus \mathbf{Z}_{2}$.

4. Complete reducibility. Let $k$ be a field, $G$ a group, and let $V_{1}$ and $V_{2}$ be $k G$ modules, finite dimensional over $k$. Then $G$ acts on $\operatorname{Hom}_{k}\left(V_{1}, V_{2}\right)$ by $(g \cdot f)(v)$ $=g\left(f\left(g^{-1} v\right)\right)$, where $g \in G, f \in \operatorname{Hom}_{k}\left(V_{1}, V_{2}\right)$ and $v \in V_{1}$. We recall that $H^{1}\left(G, \operatorname{Hom}_{k}\left(V_{2}, V_{1}\right)\right)$ is in one-one correspondence with the (equivalence classes of) extensions of $V_{1}$ by $V_{2}$ [5, Chapter 10, Theorem 10], and the zero element corresponds to the split extension. 
COROLlary 4. Let $V$ be a finite-dimensional vector space over a field $k$ and let $G$ be a subgroup of $G L(V)$. If $G$ contains a subnormal abelian subgroup $N$ such that the centralizer of $N$ in End $V$ contains no nonzero nilpotent elements, then $G$ is completely reducible.

Proof. Let $W$ be a $G$-invariant subspace of $V$. We must show that the following exact sequence of $G$-spaces splits: $0 \rightarrow W \rightarrow V \rightarrow V / W \rightarrow 0$. By the remarks above, together with Theorem 1 , it will be sufficient to show that $\operatorname{Hom}_{k}(V / W, W)^{N}=0$. Let $f^{\prime} \in \operatorname{Hom}_{k}(V / W, W)^{N}$. Then, the composite $f=i \circ f^{\prime} \circ \nu$, where $\nu: V \rightarrow V / W$ is the natural map and $i: W \rightarrow V$ is inclusion, is in the centralizer of $N$ in End $V$, and $f^{2}=0$, so $f=0$. Thus $f^{\prime}=0$.

Note that we can extract a more general statement from the proof of Corollary 4. Namely, if we replace the hypothesis "no nilpotent in the centralizer of $N$ " by "no nilpotent $f$ in the centralizer of $N$ such that $f(V) \subset W$ ", then we may conclude that $W$ has an invariant complement. For, the endomorphism $f$ constructed in the proof clearly satisfies the forbidden condition, hence is 0 , so $f^{\prime}=0$.

\section{REFERENCES}

1. H. Cartan and S. Eilenberg, Homological algebra, Princeton Univ. Press, Princeton, N.J., 1956. MR 17, 1040.

2. K. W. Gruenberg, Cohomological topics in group theory, Lecture Notes in Math., vol. 143, Springer-Verlag, Berlin and New York, 1970. MR 43 \#4923.

3. N. Jacobson, Lectures in abstract algebra. Vol. II: Linear algebra, Van Nostrand, Princeton, N.J., 1953. MR 14, 837.

4. S. Lang, Algebra, Addison-Wesley, Reading, Mass., 1965. MR 33 \#5416.

5. D. G. Northcott, An introduction to homological algebra, Cambridge Univ. Press, New York, 1960. MR 22 \#9523.

6. J.-P. Serre, Corps locaux, Publ. Inst. Math. Univ. Nancago, VIII, Actualités Sci. Indust., no. 1296, Hermann, Paris, 1962. MR 27 \# 133.

Department of Mathematics, Fordham University, Bronx, New York 10458 